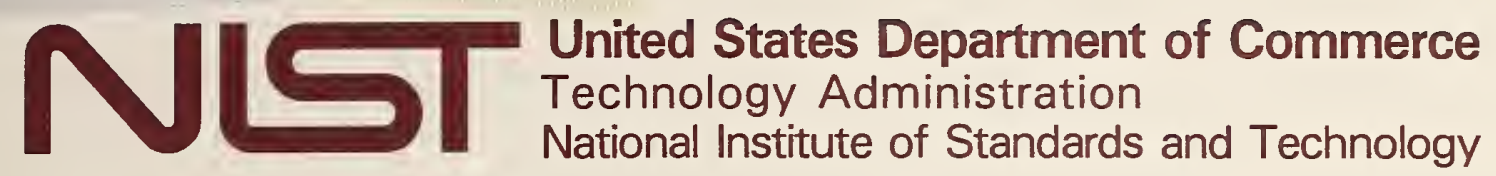

NIST Technical Note 1379

\title{
Direct Comparison Transfer of Microwave Power Sensor Calibrations
}

M.P. Weidman

QC

100

.05753

N0.1379 



\section{Direct Comparison Transfer of Microwave Power Sensor Calibrations}

M.P. Weidman

Electromagnetic Fields Division

Electronics and Electrical Engineering Laboratory

National Institute of Standards and Technology

325 Broadway

Boulder, Colorado 80303-3328

January 1996

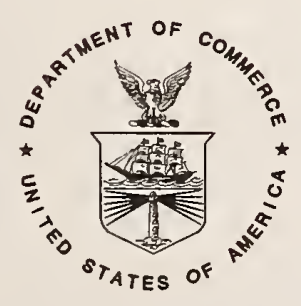

U.S. DEPARTMENT OF COMMERCE, Ronald H. Brown, Secretary TECHNOLOGY ADMINISTRATION, Mary L. Good, Under Secretary for Technology NATIONAL INSTITUTE OF STANDARDS AND TECHNOLOGY, Arati Prabhakar, Director 
National Institute of Standards and Technology Technical Note Natl. Inst. Stand. Technol., Tech. Note 1379, 20 pages (January 1996) CODEN:NTNOEF

\section{U.S. GOVERNMENT PRINTING OFFICE \\ WASHINGTON: 1996}

For sale by the Superintendent of Documents, U.S. Government Printing Office, Washington, DC 20402-9325 


\section{Contents}

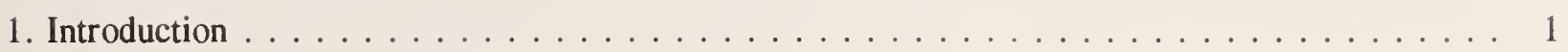

2. Basic Theory ................................. 3

2.1 Efficiency Definitions . . . . . . . . . . . . . . . . 3

2.2 Transfer without Directional Coupler .................... 3

2.3 Transfer with Directional Coupler . . . . . . . . . . . . . . . . 4

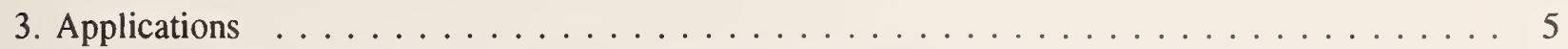

3.1 Measurement of $\Gamma_{\mathrm{G}}$ without a Directional Coupler $\ldots \ldots \ldots \ldots$

3.2 Measurement of $\Gamma_{\mathrm{G}}$ of a Directional Coupler . . . . . . . . . . . . 6

3.3. Other Types of Directional Couplers . . . . . . . . . . . . . . . . 7

3.3 .1 VSWR Bridge $\ldots \ldots \ldots \ldots \ldots \ldots \ldots \ldots \ldots \ldots \ldots \ldots \ldots \ldots \ldots$

3.3.2 Resistive Power Splitter . . . . . . . . . . . . . . . . 8

5. Uncertainty Analysis $\ldots \ldots \ldots \ldots \ldots \ldots \ldots \ldots$

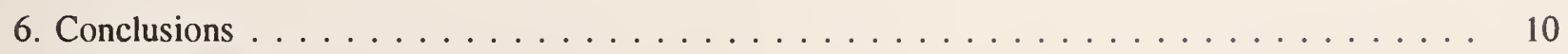

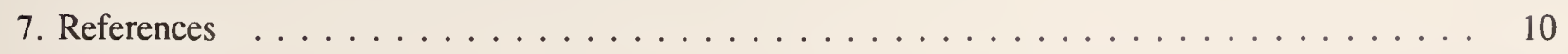

Appendix A. Uncertainty in Mismatch Factor . . . . . . . . . . . . . . . . . 12 



\title{
DIRECT COMPARISON TRANSFER OF MICROWAVE POWER SENSOR CALIBRATIONS
}

\author{
M. P. Weidman \\ National Institute of Standards and Technology \\ Boulder, Colorado 80303
}

\begin{abstract}
This report describes a basic, but potentially accurate, transfer technique for comparing microwave power sensors. The technique is not new, but the specific applications are. This report is written to supplement the existing literature. The method transfers the effective efficiency of a standard power sensor to an unknown (uncalibrated) power sensor. The power sensors may be bolometric (thermistor mounts), thermoelectric, or diode types, and each type will have inherent limitations. The technique can be implemented with a variety of commercial coaxial and rectangular waveguide components. Measurement uncertainty is discussed in this report so that a potential user can quantify transfer uncertainties.
\end{abstract}

Key words: direct comparison; effective efficiency; microwave metrology; mismatch uncertainty; power sensor; power meter; power transfer

\section{Introduction}

This paper shows how to use direct comparison over broad frequency ranges with low uncertainties. In this report, the generic term, power sensor is used to describe several types of microwave sensor in common use. Although the most nearly linear power sensor is the thermistor mount, thermoelectric and diode sensors can also be used (with limitations). All sensors are used with some type of instrumentation or power meter indicator. The indicated power is proportional to the net radio frequency ( $\mathrm{rf}$ ) or microwave power absorbed by the sensor. The purpose of calibration of the sensor is to determine this proportionality.

The direct comparison measurement technique is based on alternate connections of a standard and unknown power meter to a source of rf or microwave power (figure 1). The source can be a signal 
generator, a generator with an isolator or attenuator, or may include a sensor-directional coupler monitor at the generator output. Direct comparison is one of the oldest and most basic of microwave power transfer techniques. References describing this technique as a calibration method date back to 1953 [1]. Other references describe different aspects of this method [2,3]. A good reference for the general topic of power comparison techniques is Part C of [4]. Other calibration techniques, such as the use of the sixport [5] or tuned reflectometers [6], are more complex and are not discussed in detail here.

One of the main sources of uncertainty in any power transfer is caused by the impedance mismatch between the source and the power sensors connected to the source. Figure 1 shows the alternate connection of two sensors to a source. The power sensors in a direct comparison are of the terminating type and usually consist of a standard sensor and one or more unknown sensors. Historically, the direct comparison method was used for single frequency or narrow-band measurements or for measurements where a low uncertainty was not required. The reasons for using the direct comparison instead of other methods are speed and simplicity. There are several options for using the technique depending on the accuracy required. The higher accuracy

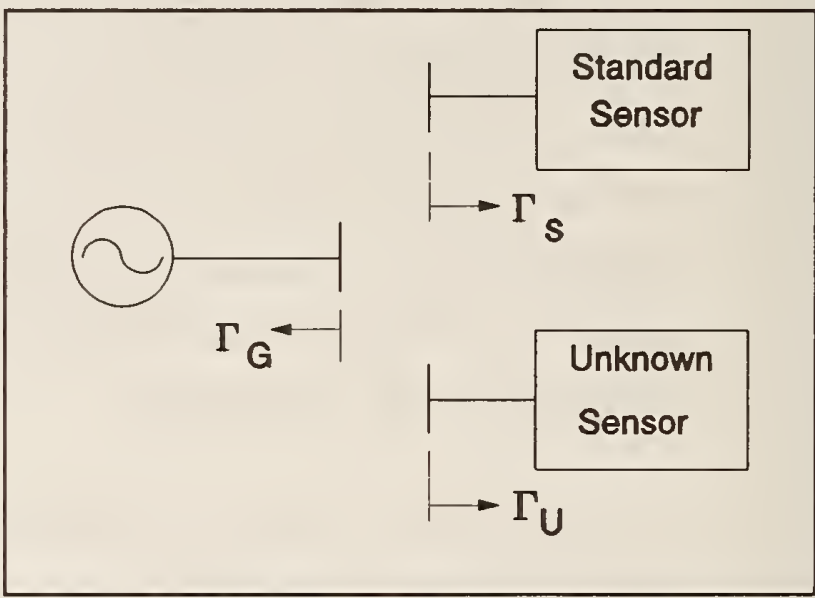

Figure 1. Alternate connection of standard and unknown detectors to a generator. implementation uses high directivity waveguide directional couplers, or in the case of coaxial sensors, VSWR bridges. Full evaluation of the uncertainty in the direct comparison technique requires separate measurements of the complex reflection coefficients $\Gamma_{S}, \Gamma_{G}$, and $\Gamma_{U}$. These reflection coefficients apply to the power standard sensor, the equivalent generator, and the device under test (DUT) sensor. The reflection coefficient measurements can be determined using a commercial vector automatic network analyzer (VANA) with appropriate speed and accuracy. 


\section{Basic Theory}

\subsection{Efficiency Definitions}

The calibration quantity used to characterize terminating power meters is effective efficiency $\eta_{E}$ for thermistor sensors and efficiency factor $\eta$ for the other types of sensors. Efficiency $\eta_{E}$ is defined as ${ }^{P_{D C}}{ }^{/ P_{R F}}$, where $P_{D C}$ is the substituted dc power and $P_{R F}$ is the net radio frequency (rf) or microwave power dissipated in the sensor. Substituted dc power is the change in direct current (dc) or low frequency power necessary to maintain the thermistor element or elements at a constant resistance when rf or microwave power is applied to the sensor.

Thermoelectric sensors convert the absorbed rf power to heat, then to dc, and instrumentation amplifies the low level dc and displays the result. Diode sensor-power meters convert rf voltage to dc voltage and display the result. In the square law region, the diode sensor-meter displays a response proportional to rf power (voltage squared). The efficiency factor $\eta$ of thermoelectric or diode sensors is $P_{M} / P_{R F}$, where $P_{M}$ is the indicated or displayed power meter reading and $P_{R F}$ is the net rf or microwave power dissipated in the sensor.

\subsection{Transfer without Directional Coupler}

Effective efficiency $\eta_{E}$ from a standard thermistor ( $\eta$ for other sensors) to a DUT sensor is transferred using

$$
\eta_{E U}=\eta_{E S} \times P_{R A T I O} \times M M
$$

where $\eta_{E U}$ is the effective efficiency of the DUT and $\eta_{E S}$ is that of the standard. When the power sensors are not thermistors, effective efficiency is replaced by efficiency defined in eq (1). P RATIO is the ratio of two substituted dc or indicated powers,

$$
P_{R A T I O}=\frac{\left(P_{D C}\right)_{U}}{\left(P_{D C}\right)_{S}}
$$


where $P(D C)_{S}$ is the dc power in the standard and $P(D C)_{U}$ is the dc power in the DUT. For thermoelectric or diode sensors, the $P_{D C}$ are replaced by the indicated powers $P_{M}$. The mismatch factor $M M$ is

$$
M M=\frac{\left(1-\left|\Gamma_{s}\right|^{2}\right)}{\left(1-\left|\Gamma_{u}\right|^{2}\right)} \times \frac{\left|1-\Gamma_{u} \Gamma_{g}\right|^{2}}{\left|1-\Gamma_{s} \Gamma_{g}\right|^{2}},
$$

where $\Gamma_{U}, \Gamma_{S}$, and $\Gamma_{G}$ are the respective complex reflection coefficients of the DUT, the standard, and the generator. Application details are described in section 3.

\subsection{Transfer with Directional Coupler}

A source which includes a generator and directional coupler-sensor on its output has several desirable features. Figure 2 shows the coupler system. The directional coupler samples the incident power from the generator and can be used for power leveling. Recording the side arm and main arm powers simultaneously is the same as leveling. Another feature of the coupler system is that $\Gamma_{G}$ at the output of the coupler is a function of only the coupler parameters and not of the generator driving the coupler [2].

A given application may include a waveguide coupler, a voltage standing wave ratio (VSWR) bridge, a power splitter, a quadrature hybrid, or some other type of directional coupling device. In a typical system, a power sensor is permanently attached to the incident-side-arm of the coupler.

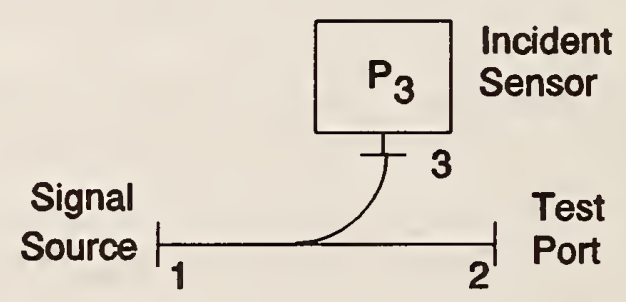

Figure 2. Directional coupler port numbering.

Effective efficiency $\eta_{E}$ from a standard thermistor

to a DUT with a directional coupler is transferred with eq (1). When the power sensors are not thermistors, effective efficiency is replaced by the efficiency factor $\eta$. Let $R$ be the ratio of substituted dc powers, $P_{S I D E} / P_{M A I N}$, where $P_{S I D E}$ is the dc power in the sensor on the side arm of the coupler, and $P_{\text {MAIN }}$ is the dc power in the power sensor on the main arm. In addition, let $R_{S}$ be that ratio with 
the standard connected and $R_{U}$ be the ratio with the DUT connected. Then a new $P_{R A T I O}$ is

$$
P_{\text {RATIO }}=\frac{R_{S}}{R_{U}},
$$

which involves four dc powers. It is not necessary to control the power level of the source if $P_{M A I N}$ and $P_{S I D E}$ are measured simultaneously. If any of the sensors are not thermistors, the dc powers are replaced by the power meter indicated powers. Mismatch factor $M M$ is the same as shown in eq (3), where $\Gamma_{U}$ and $\Gamma_{S}$ are the same, and $\Gamma_{G}$ is the complex reflection coefficient of the coupler equivalent generator. The definition of $\Gamma_{G}$ for a directional coupler comes from ref. [2] and is

$$
\Gamma_{G}=S_{22}-\frac{S_{12} S_{23}}{S_{13}}
$$

where the $S_{i j}$ are the scattering parameters (S-parameters) of the directional coupler used in the power transfer. Figure 2 shows the port numbering for the S-parameters of the coupler.

\section{Applications}

The value of $\eta_{E S}$ of a standard thermistor or $\eta$ of a standard thermoelectric (or diode) sensor must be known for a power transfer. Efficiency $\eta_{E S}$ may come from a national laboratory, a calibration laboratory, or a manufacturer.

The other information necessary for an accurate power transfer is $M M$. The complex values of $\Gamma_{G}, \Gamma_{S}$, and $\Gamma_{U}$ are required for best accuracy. If it is possible to measure only the magnitude of the reflection coefficients, then there is an uncertainty in percent in $M M$ of

$$
\Delta(M M)=200\left(\left|\Gamma_{G}\right|\left|\Gamma_{S}+\right| \Gamma_{G}|| \Gamma_{U} \mid\right)
$$

For typical values of $\Gamma_{G}, \Gamma_{S}$, and $\Gamma_{U}$, the uncertainty in $M M$ can range from 1 percent to 15 percent if only magnitudes of $\Gamma$ are measured. Equation (6) comes from a first-order approximation to the derivative of eq (3). 


\subsection{Measurement of $\Gamma_{G}$ without a Directional Coupler}

$\Gamma_{G}$ for an active source is difficult to measure, although a technique has been suggested if the source can tolerate high reflected power $[7,8]$. A better way to use a source without the directional coupler is to put an isolator or attenuator on its output. If low power is a problem, then the isolator would be preferable, but isolators are limited in frequency range.

An approximation to $\Gamma_{G}$ with an attenuator or isolator on the output of the generator is $\Gamma$ looking back into the output of the attenuator or isolator. This $\Gamma$ can be measured without the generator turned on. The uncertainty of this measurement depends on the $\Gamma$ looking back into the active generator and the amount of attenuation or isolation. Every $10 \mathrm{~dB}$ of attenuation or $20 \mathrm{~dB}$ of isolation will reduce the effect of the active source $\Gamma$ by an order of magnitude. The approximation to $\Gamma_{G}$ is due to the fact that the measurement looking back into the attenuator (or isolator), toward the generator, is performed with the generator off and is typically negligible.

As an example, for an active generator with $\Gamma=0.3 \pm 0.3$ and $20 \mathrm{~dB}$ of attenuation or $40 \mathrm{~dB}$ of isolation, the uncertainty in $\Gamma_{G}$ would be 0.003 . The estimate of uncertainty for the generator $\Gamma_{G}( \pm 0.3)$ applies to most known signal generators. The approximation uncertainty in $\Gamma_{G}$ must be propagated through $M M$. The propagation of uncertainty in $M M$ due to uncertainties in reflection coefficient is analyzed in Appendix A.

\subsection{Measurement of $\Gamma_{G}$ of a Directional Coupler}

The definition of $\Gamma_{G}$ is given in eq (5). $\Gamma_{G}$ is a function of the S-parameters of the coupler only and is not affected by the generator. Reference [2] describes one way to measure $\Gamma_{G}$, but this method requires a tuning adjustment at each measurement frequency.

An approximate measurement method, which can be done over a broad frequency range with a vector network analyzer, is to measure only the $S_{22}$ term for the coupler. The second term in eq (5) involves the directivity $20 * \log \left(\left|S_{23}\right| /\left|S_{13}\right|\right)$ and the main line loss $20 * \log \left(\left|S_{12}\right|\right)$. S-parameters are defined as voltage ratios, so that decibel $(\mathrm{dB})$ values are $20 * \log (S)$. The second term on the right side of eq (5) can be ignored if the directivity is high. The magnitude of the directivity term is shown in the examples. 
$S_{22}$ is the $\Gamma$ looking into port 2 (fig. 2) with the other ports terminated in nonreflecting loads. There are three elements to the uncertainty in $\Gamma_{G}$ when approximating it by $S_{22}$ :

(1) The finite directivity of the coupler or bridge.

(2) The assumption of a matched load on port 1 when measuring $\Gamma$ at port 2 . Attenuation between port 1 and port 2 reduces the effect of not having a matched load on port 1 by $\left|S_{12}\right| *\left|S_{21}\right|$. This is the same order of magnitude reduction for each $10 \mathrm{~dB}$ of attenuation as described in section 2.1.

(3) An imperfect load on port 3.

Some examples should clarify the measurement of $\Gamma_{G}$. If the directivity of the coupler is $40 \mathrm{~dB}$, the uncertainty in $\left|\Gamma_{G}\right|$ from element (1) is 0.01 . With an isolator of $20 \mathrm{~dB}$, or a $10 \mathrm{~dB}$ attenuator on port 2, (along with $40 \mathrm{~dB}$ directivity) the uncertainty in $\left|\Gamma_{G}\right|$ due to element (1) would be 0.001 , which is negligible for most purposes.

For a $3 \mathrm{~dB}$ coupler (without an attenuator or isolator) with a 0.02 load on port 1, element (2) would result in an uncertainty of 0.01 . A better condition would be to add a $10 \mathrm{~dB}$ pad or $20 \mathrm{~dB}$ isolator on port 2 , then element (2) would result in an uncertainty of 0.002 .

Element (3), which is a function of the load on port 3 involves the two-way loss from port 2 to port 3 and for a $40 \mathrm{~dB}$ coupler directivity and $3 \mathrm{~dB}$ forward coupling, the effect of the $\Gamma$ on port 3 is reduced by 0.00005 . Even a short circuit on port 3 has little effect on $S_{22}$.

All of the uncertainties in $\Gamma_{G}$ must be propagated through $M M$ to get the uncertainty in the power transfer, which is described in Appendix A. The previous examples should help explain how to calculate an uncertainty in $M M$ for different combinations of hardware.

\subsection{Other Types of Directional Couplers}

Figure 2 and the general description so far correspond to waveguide directional couplers. Other types of couplers are useful for the direct comparison power transfer method.

\subsubsection{VSWR Bridge}

Figure 3 shows the rf hardware for one possible coaxial power calibration system. The VSWR bridge 
is the same as the directional coupler. The VSWR bridge was originally designed for measuring VSWR with the generator on arm 2, the test port on arm 1 and a sensor on arm 3. The application of this device to a power transfer is a new innovation. The $3 \mathrm{~dB}$ attenuator on arm 2 of the bridge provides $6 \mathrm{~dB}$ of return loss isolation for the $S_{22}$ measurement. $S_{22}$ is defined with matched loads on all other ports. In the example of figure 3 , a nonideal load with $\Gamma=0.02$ connected to port 1 , when measuring $S_{22}$, will

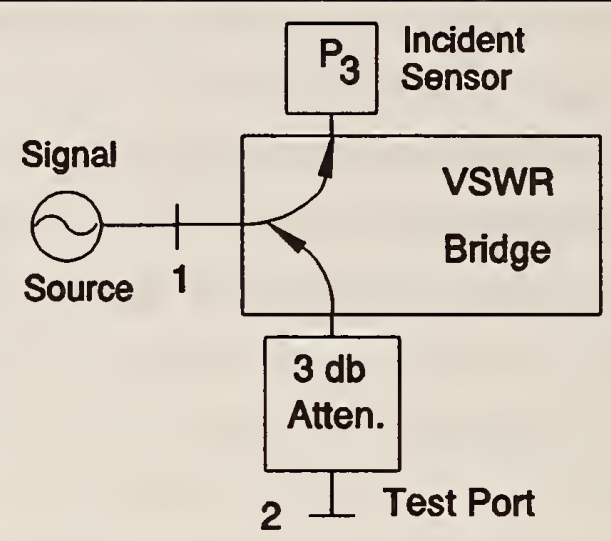

Figure 3. VSWR bridge used as directional coupler for power transfer.

create an uncertainty in $S_{22}$ of 0.02 times 0.1259 or 0.0025 . The 0.1259 term comes from the two-way loss through the sum of the $3 \mathrm{~dB}$ attenuator and the $6 \mathrm{~dB}$ coupling inherent to the VSWR bridge ( $9 \mathrm{~dB}$ corresponds to a voltage ratio of 0.1259$)$. The uncertainty due to finite directivity is $\left|S_{23} * S_{12}\right| /\left|S_{13}\right|$ or $\mathbf{0 . 0 0 3 5 5}$. The uncertainty due to finite directivity, for this example, is based on a directivity $\left(\left|S_{23} / S_{13}\right|\right)$ of $40 \mathrm{~dB}$ plus the $9 \mathrm{~dB}$ loss from port 1 to port 2 (6 dB coupling $+3 \mathrm{~dB}$ attenuator).

\subsubsection{Resistive Power Splitter}

Another type of coaxial directional coupler is the resistive power splitter shown schematically in figure 4. This device has a nominal coupling of $6 \mathrm{~dB}$ between ports 1 and 2 and ports 1 and 3 . If this device is perfectly symmetrical with no parasitic reactances, $S_{22}=0.25, S_{23}=0.25$, and $S_{13}=S_{12}$, so $\Gamma_{G}$ from eq (5) is zero. $S_{22}$ for a power splitter is not a good approximation to $\Gamma_{G}$, so even though $\Gamma_{G}$ may be small, a measurement of its value is difficult but necessary for a thorough uncertainty analysis. One way to measure $\Gamma_{G}$ is to use a VANA to measure all the complex S-parameters in eq (5) and calculate $\Gamma_{G}$. A slight modification to this takes advantage of the approximation, $S_{21}=S_{31}$, so that from eq (5) $\Gamma_{G}=S_{22}-S_{23}$, but this method still requires

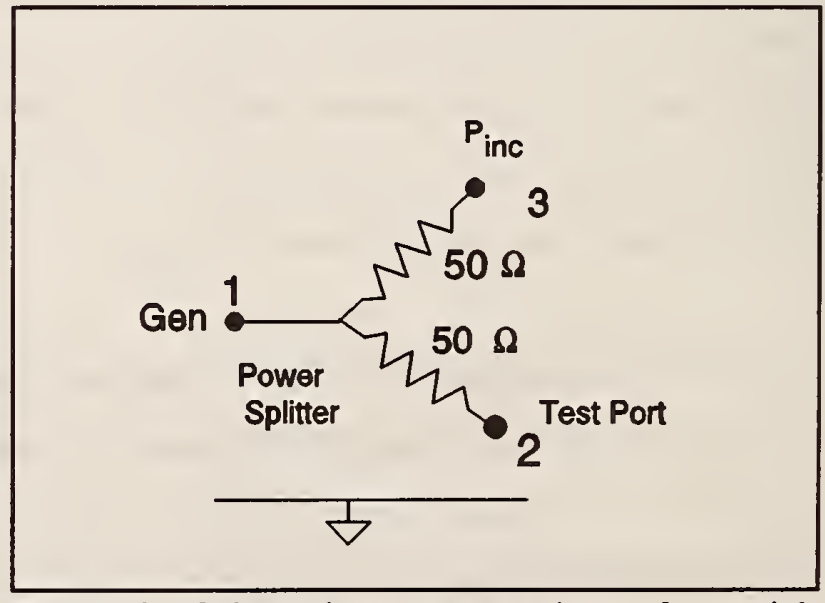

Figure 4. Schematic representation of coaxialresistive power splitter. 
measurement of complex S-parameters. A third way is to use a sliding short coupled to a slotted line $[7,8]$, but since this is a single frequency method, it would be just as easy to use the method described in ref. [2]. A comparison of the second and third methods for measuring $\Gamma_{G}$ on power splitters show a range of uncertainties of 0.01 to 0.03 in $\Gamma_{G}$ [9]. As in the preceding sections, any uncertainty in $\Gamma_{G}$ must be propagated through $M M$ (eq (3)) to determine the uncertainty in the power transfer.

\section{Uncertainty Analysis}

This section is intended as a general guide in determining the uncertainty for a power transfer using the techniques described in the previous sections. The method of combining uncertainties, root sum of squares (RSS), for example, or probability considerations (coverage factors) are left to the user.

The uncertainty in measuring $\eta_{E S}$ for an unknown device (DUT) is a combination of the uncertainties in the elements in eqs (1) to (3). The following list of uncertainties can be used as a guideline.

\section{Power Standard $\eta_{E S}$ Uncertainty:}

The first element on the right side of eq (1) is the effective efficiency $\eta_{E S}$ of the standard thermistor mount. The value of this uncertainty normally comes from the standards or calibration laboratory which provides the efficiency for the working standard.

\section{$P_{\text {ratio }}$ Uncertainty:}

Sources of uncertainty include, but are not limited to, power and frequency instability and connector repeatability. Instrumentation uncertainties such as resolution or offsets also occur here. These uncertainties are determined experimentally, using the actual hardware, by repeating the process and calculating the variability (standard deviation).

\section{Mismatch Factor Uncertainty:}

Mismatch factor $M M$ in eq (3) has an uncertainty based on the VANA measurement of the reflection coefficients $\Gamma$ and the approximations described in the basic theory above. The VANA uncertainty is dependent on the calibration technique, for example, sliding load or TRL. Equation (3) shows that the effect of the $\Gamma$ measurement on the power measurement is of second order. The differential of eq (3), expressed as partial derivatives, can be used to determine the uncertainty in the power transfer. Appendix A gives the equations. 


\section{Conclusions}

Several relatively simple calibration procedures which yield a power sensor transfer with an uncertainty which can be fully characterized have been described. The transfer system can be constructed from commercially available hardware.

The author thanks Fred Clague and Marilyn Packer for their encouragement and suggestions. Thanks also to Claude Weil and Thomas Scott for their suggestions.

\section{References}

[1] Beatty, R.W.; Macpherson, A.C. Mismatch Errors in Microwave Power Measurements. Proc. IRE, 41(9): 1112-1119; 1953 September.

[2] Engen, G.F. Amplitude Stabilization of a Microwave Signal Source. IRE Trans. Microwave Theory Tech., MTT-6(2): 202-206; 1958 April.

[3] Desch, R.F.; Larson, R.E. Bolometric Microwave Power Calibration Techniques at the National Bureau of Standards. IEEE Trans. Instrum. Meas., IM-12(1): 29-33; 1963 June.

[4] Fantom, A.E.; Radio Frequency and Microwave Power Measurement. Peter Perigrinus Ltd., London, United Kingdom, 1990.

[5] Engen, G.F.; Hoer, C.A. Application of an Arbitrary 6-port Junction to Power Measurement Problems. IEEE Trans. Instrum. Meas., IM-21(4): 470-474; 1972 November.

[6] Engen, G.F. A Transfer Instrument for the Intercomparison of Microwave Power Meters. IRE Trans. Instrum., IM-9: 202-208; 1960 September.

[7] Sorger, G.U.; Weinschel, B.O.; technique presented at IMEKO Symposium on Microwave Measurements; October, 1966. 
[8] Alford, A. Measurement of Generator Impedance Using a Sliding Short Circuit. Microwave J., 29(8): 117-118; 1986 November.

[9] Ulriksson, B.A.; Lucas Weinschel internal report. Lucas Weinschel, Gaithersburg, Maryland. 


\section{Appendix A. Uncertainty in Mismatch Factor}

This appendix shows the derivation of the equations necessary to calculate the uncertainty in $M M$ defined in the Basic Theory section 2.

Equation (3) is repeated here as eq (A1),

$$
M M=\frac{\left(1-\left|\Gamma_{S}\right|^{2}\right)\left|1-\Gamma_{G} \Gamma_{U}\right|^{2}}{\left(1-\left|\Gamma_{U}\right|^{2}\right)\left|1-\Gamma_{G} \Gamma_{S}\right|^{2}}
$$

where the mismatch factor is $M M$. The differential of $M M$ is

$$
d(M M)=\frac{\partial(M M)}{\partial\left|\Gamma_{G}\right|} d\left|\Gamma_{G}\right|+\frac{\partial(M M)}{\partial\left|\Gamma_{S}\right|} d\left|\Gamma_{S}\right|+\frac{\partial(M M)}{\partial\left|\Gamma_{U}\right|} d\left|\Gamma_{U}\right|+\frac{\partial(M M)}{\partial \Phi_{G S}} d \Phi_{G S}+\frac{\partial(M M)}{\partial \Phi_{G U}} d \Phi_{G U}
$$

where the partials are with respect to the magnitudes of the three $\Gamma s$, and $\Phi_{G S}$, and $\Phi_{G U}$ are defined by

$$
\begin{aligned}
& \phi_{G S}=\phi_{G}+\phi_{S}, \\
& \phi_{G U}=\phi_{G}+\phi_{U},
\end{aligned}
$$

where $\Phi_{G}, \Phi_{S}$, and $\Phi_{U}$ are the phase angles of $\Gamma_{G}, \Gamma_{S}$, and $\Gamma_{U}$.

In order to simplify the algebra, the second ratio in $M M$ in eq (A1) was changed to

$$
\frac{\left|1-\Gamma_{G} \Gamma_{U}\right|^{2}}{\left|1-\Gamma_{G} \Gamma_{S}\right|^{2}}=\frac{1-2\left|\Gamma_{G} \Gamma_{U}\right| \cos \left(\Phi_{G}+\Phi_{U}\right)+\left|\Gamma_{G} \Gamma_{U}\right|^{2}}{1-2\left|\Gamma_{G} \Gamma_{S}\right| \operatorname{Cos}\left(\Phi_{G}+\Phi_{S}\right)+\left|\Gamma_{G} \Gamma_{S}\right|^{2}}
$$

This simplification gets rid of the complex terms in eq (A1) and leads to the use of $\Phi_{G S}$ and $\Phi_{G U}$ in 
eq (A2).

Equations (A6) through (A10) follow from eqs (A2) through (A5).

$$
\begin{gathered}
\frac{\partial(M M)}{\partial\left|\Gamma_{G}\right|}=2\left[\frac{(M M d) B\left(1-C^{2}\right)(A B-D)-(M M n) C\left(1-B^{2}\right)(A C-E)}{(M M d)^{2}}\right], \\
\frac{\partial(M M)}{\partial\left|\Gamma_{U}\right|}=2\left[\frac{A\left(1-C^{2}\right)(A B-D)+(M M n) B /\left(1-B^{2}\right)}{(M M d)}\right], \\
\frac{\partial(M M)}{\partial\left|\Gamma_{s}\right|}=-2(M M n)\left[\frac{A\left(1-B^{2}\right)(A C-E)+(M M d) C /\left(1-C^{2}\right)}{(M M d)^{2}}\right] \\
\frac{\partial(M M)}{\partial(D)}=\frac{-2 A B\left(1-C^{2}\right)}{(M M d)} \\
\frac{\partial(M M)}{\partial(E)}=\frac{2(M M n) A C\left(1-B^{2}\right)}{(M M d)^{2}} .
\end{gathered}
$$

Definitions for the shorthand terms in eqs (A6) through (A10) are

$$
\begin{gathered}
M M n=\text { numerator of } M M \text { ratio, } \\
M M d=\text { denominator of } M M \text { ratio, } \\
A=\left|\Gamma_{G}\right|, B=\left|\Gamma_{U}\right|, \text { and } C=\left|\Gamma_{S}\right|, \\
D=\operatorname{COS}\left(\phi_{G}+\phi_{U}\right), \text { and } E=\operatorname{CoS}\left(\phi_{G}+\phi_{S}\right) .
\end{gathered}
$$


The phase angle uncertainties which are needed along with eqs (A9) and (A10) are defined in eq (A15). The partial derivative of $M M$ with respect to $D$ and $E$ are multiplied by $d(D)$ and $d(E)$ and then replace the last two terms in eq (A2):

$$
d(D)=-\operatorname{SIN}\left(\phi_{G U}\right) d\left(\phi_{G U}\right) \text { and } d(E)=-\operatorname{SIN}\left(\phi_{G S}\right) d\left(\phi_{G S}\right) \text {, }
$$

where $d(\Phi)$ is the uncertainty in $\Phi_{G S}$ and $\Phi_{G U}$ in radians. In reflection coefficient measurements, the uncertainty in the phase of $\Gamma$ is inversely proportional to the magnitude of $\Gamma$. As the magnitude of $\Gamma$ approaches zero, the uncertainty in the phase of $\Gamma$ approaches $\pm \pi \mathrm{rad}$, but the product $A B$ in the numerator of eq (A9), or $A C$ in the numerator of (A10) approaches zero at the same time, so the partial derivatives of $M M$ times the differentials, $d(\Phi)$, approach zero.

Equations (A6) through (A15) are used in the calculation of the uncertainty in the power transfer caused by uncertainties in the reflection coefficient measurements. 


Journal of Research of the National Institute of Standards and Technology-Reports NIST research and development in those disciplines of the physical and engineering sciences in which the Institute is active. These include physics, chemistry, engineering, mathematics, and computer sciences. Papers cover a broad range of subjects, with major emphasis on measurement methodology and the basic technology underlying standardization. Also included from time to time are survey articles on topics closely related to the Institute's technical and scientific programs. Issued six times a year.

\section{Nonperiodicals}

Monographs-Major contributions to the technical literature on various subjects related to the Institute's scientific and technical activities.

Handbooks-Recommended codes of engineering and industrial practice (including safety codes) developed in cooperation with interested industries, professional organizations, and regulatory bodies.

Special Publications-Include proceedings of conferences sponsored by NIST, NIST annual reports, and other special publications appropriate to this grouping such as wall charts, pocket cards, and bibliographies.

Applied Mathematics Series-Mathematical tables, manuals, and studies of special interest to physicists, engineers, chemists, biologists, mathematicians, computer programmers, and others engaged in scientific and technical work.

National Standard Reference Data Series-Provides quantitative data on the physical and chemical properties of materials, compiled from the world's literature and critically evaluated. Developed under a worldwide program coordinated by NIST under the authority of the National Standard Data Act (Public Law 90-396). NOTE: The Journal of Physical and Chemical Reference Data (JPCRD) is published bimonthly for NIST by the American Chemical Society (ACS) and the American Institute of Physics (AIP). Subscriptions, reprints, and supplements are available from ACS, 1155 Sixteenth St., NW, Washington, DC 20056.

Building Science Series-Disseminates technical information developed at the Institute on building materials, components, systems, and whole structures. The series presents research results, test methods, and performance criteria related to the structural and environmental functions and the durability and safety characteristics of building elements and systems.

Technical Notes-Studies or reports which are complete in themselves but restrictive in their treatment of a subject. Analogous to monographs but not so comprehensive in scope or definitive in treatment of the subject area. Often serve as a vehicle for final reports of work performed at NIST under the sponsorship of other government agencies.

Voluntary Product Standards-Developed under procedures published by the Department of Commerce in Part 10, Title 15, of the Code of Federal Regulations. The standards establish nationally recognized requirements for products, and provide all concerned interests with a basis for common understanding of the characteristics of the products. NIST administers this program in support of the efforts of privatesector standardizing organizations.

Consumer Information Series-Practical information, based on NIST research and experience, covering areas of interest to the consumer. Easily understandable language and illustrations provide useful background knowledge for shopping in today's technological marketplace.

Order the above NIST publications from: Superintendent of Documents, Government Printing Office, Washington, DC 20402.

Order the following NIST publications-FIPS and NISTIRs-from the National Technical Information Service, Springfield, VA 22161.

Federal Information Processing Standards Publications (FIPS PUB)-Publications in this series collectively constitute the Federal Information Processing Standards Register. The Register serves as the official source of information in the Federal Government regarding standards issued by NIST pursuant to the Federal Property and Administrative Services Act of 1949 as amended, Public Law 89-306 (79 Stat. 1127), and as implemented by Executive Order 11717 (38 FR 12315, dated May 11, 1973) and Part 6 of Title 15 CFR (Code of Federal Regulations).

NIST Interagency Reports (NISTIR)-A special series of interim or final reports on work performed by NIST for outside sponsors (both government and non-government). In general, initial distribution is handled by the sponsor; public distribution is by the National Technical Information Service, Springfield, VA 22161 , in paper copy or microfiche form. 
U.S. Department of Commerce

National Institute of Standards and Technology

325 Broadway

Boulder, Colorado 80303-3328

\section{Official Business}

Penalty for Private Use, $\$ 300$ 